\title{
Qualidade do almoço de trabalhadores segundo o Programa de Alimentação dos Trabalhadores e o Índice de Qualidade da Refeição
}

\author{
Maria Sônia Lopes Duarte ${ }^{1}$, Lisiane Lopes da Conceição², Luíza Carla Vidigal Castro ${ }^{3}$, \\ Eliana Carla Gomes de Souza ${ }^{4}$
}

O objetivo dessa pesquisa foi avaliar a qualidade do almoço de uma Unidade de Alimentação e Nutrição (UAN) segundo as recomendações do Programa de Alimentação do Trabalhador (PAT) e o Índice de Qualidade da Refeição. Foi realizado um estudo transversal em uma UAN de uma empresa privada, cadastrada no PAT e com supervisão de um nutricionista. A coleta de dados ocorreu em três dias não consecutivos, para todas as preparações servidas no almoço. A avaliação do percentual de adequação de energia, sódio, macronutrientes, NDPcal\% e fibras excederam as recomendações estabelecidas pelo PAT, enquanto que para o carboidrato o almoço forneceu quantidades abaixo da recomendação. Para o Índice de Qualidade da Refeição, a média encontrada foi de 56,53 pontos e a classificação da refeição foi de que precisa de melhorias. O almoço oferecido pela UAN não estava adequado de acordo com os parâmetros do PAT e o Índice de Qualidade da Refeição. A alimentação oferecida pela empresa avaliada estava inadequada nutricionalmente e, assim, torna-se necessária a inclusão de ações de correções no planejamento do cardápio bem como de medidas educativas de intervenção alimentar.

Palavras-chave: alimentação coletiva, Dietética, trabalhadores.

\section{Quality of lunch of workers according to the worker's food program and the Meal Quality Index}

This objective of this research was assessed the quality of the lunch offered in the Food and Nutrition Units in accordance with the recommendations of the Worker's Food Program (WFP) of the Meal Quality Index. Crosssectional study in Nutrition and Food Service to a private, registered in the WFP which is supervised by a nutritionist. The data's collection occurred in three non-consecutive days, for all preparations served at lunch. The results showed that the consumption of energy, sodium, macronutrients, NDPcal $\%$ and fibers by individuals studied exceeded the recommendations established by WFP, while for carbohydrate lunch provided quantities below the recommendation. For the Meal Quality Index, the average found was 56.53 points and the classification of the meal was that needs improvement. The lunch offered by the Food and Nutrition Units was not appropriate according to the parameters of the Worker's Food Program and the Meal Quality Index. The feeding offered by

1 Professora Adjunta. Departamento de Nutrição e Saúde, Universidade Federal de Viçosa. Correspondência: Av. PH Rolfs s/n, Campus Universitário, CEP: 36571-900, Viçosa, MG - Brasil. Telefone: (31) 3899 3741/ (31) 3881 8920.E-mail: msonia.duarte@ufv.br

2 Doutoranda em Ciência da Nutrição, Universidade Federal de Viçosa, Viçosa, Minas Gerais, Brasil. E-mail: lisianelopes@yahoo.com.br

${ }^{3}$ Professora Adjunta. Departamento de Nutrição e Saúde, Universidade Federal de Viçosa. E-mail: luizacvc@gmail.com

${ }^{4}$ Professora Adjunta. Departamento de Nutrição e Saúde, Universidade Federal de Viçosa. E-mail: egomesdesouza@yahoo.com.br 
the company studied was nutritionally inadequate and thus becomes necessary to include actions such as fixes in menu planning and food intervention educational measures.

Key-words: collective feeding, Dietetics, workers.

\section{INTRODUÇÃO}

O Programa de Alimentação do Trabalhador (PAT) é um programa do Ministério do Trabalho e Emprego instituído pela Lei no 6.321, de 14 de abril de 1976 e regulamentado pelo Decreto no 5, de 14 de janeiro de 1991, que prioriza o atendimento aos trabalhadores de baixa renda, isto é, aqueles que ganham até cinco salários mínimos mensais [1] O PAT beneficia mais de 15,8 milhões de trabalhadores no país e, deste total, mais de 13 milhões de trabalhadores se encaixam nessa faixa salarial. Para incentivar a adesão das empresas ao programa, o governo concede benefícios fiscais, como a dedução de até 4\% do Imposto de Renda com os gastos de alimentação. Atualmente, estão inscritas no PAT mais de 208 mil empresas [2].

O programa tem como objetivo principal melhorar as condições nutricionais dos trabalhadores, facultando às empresas a dedução de despesas com alimentação de seus funcionários. Por meio da alimentação saudável, procura-se obter repercussões positivas na qualidade de vida, redução de acidentes de trabalho e aumento da produtividade [1]. Entretanto, alguns estudos têm demonstrado que as empresas cadastradas no PAT têm ofertado uma quantidade excessiva de lipídios e energia nas refeições $[3,4,5,6]$, o que está contribuindo para um aumento na prevalência de obesidade e dislipidemias entre os trabalhadores beneficiados pelo programa $[3,4]$.

Bandoni e Jaime [7] avaliaram o Índice de Qualidade de Refeição (IQR) em 72 Unidades de Alimentação e Nutrição (UAN) de empresas beneficiárias do PAT. Os resultados mostraram que as refeições oferecidas por empresas de menor porte e estrutura apresentaram refeições com menor IQR, indicando que estas são as prioritárias para intervenções. Com o objetivo de avaliar o conhecimento dos gestores sobre promoção de alimentação saudável e saúde e a interface deste tema com o PAT, Bandoni et al. [8] realizaram um estudo com 70 empresas na cidade de São Paulo e encontraram que a maioria dos discursos dos gestores não teve ênfase na promoção da alimentação saudável e saúde.
Desta forma, para promover a alimentação saudável e, consequentemente, a saúde no ambiente de trabalho, é preciso que a alimentação oferecida pelas empresas cadastradas no PAT esteja adequada às recomendações nutricionais e contribua para a redução de doenças como a obesidade, o diabetes, as doenças cardíacas e alguns tipos de câncer [?].

O ambiente de trabalho é reconhecido como um local estratégico para promoção da saúde e alimentação saudável [10] e de acordo com a WORLD HEALTH ORGANIZATION (WHO) [11], o local de trabalho deve estimular os trabalhadores a fazer escolhas saudáveis. Para isto, as atividades desenvolvidas pelos nutricionistas nas UANs devem priorizar a qualidade nutricional das refeições e a educação nutricional dos usuários, porém, esses têm como atividade primordial a administração com ênfase no controle de custos ${ }^{[8]}$.

Diante do exposto, o presente trabalho teve como objetivo avaliar a qualidade do almoço servido a trabalhadores segundo o Programa de Alimentação do Trabalhador e o IQR.

\section{MATERIAL E MÉTODOS}

Trata-se de um estudo transversal realizado em uma Unidade de Alimentação e Nutrição (UAN) de pequeno porte, modalidade autogestão, de uma empresa privada do ramo industrial. A empresa está cadastrada no PAT e a coleta de dados foi realizada em 3 (três) dias, no período de julho a agosto de 2013. A UAN foi selecionada por conveniência, já que a pesquisa necessita do envolvimento da empresa e do nutricionista que administra o serviço de alimentação, uma vez que para a determinação das porções médias consumidas é necessário que se faça a pesagem dos alimentos nas horas de pico da produção, o que interfere no desenvolvimento do serviço.

A pesquisa foi realizada durante o almoço, em três dias não consecutivos, para todas as preparações servidas (Quadro 1). A cada dia, para a quantificação do total de preparação consumida, pesaram-se todas as preparações produzidas no horário do almoço, todas as 
sobras e os restos, em uma balança digital marca Toledo $^{\odot}$ com capacidade para $20 \mathrm{~kg}$, sensibilidade $1 \mathrm{~g}$, de acordo com Abreu et al. [12].

O peso da porção média consumida foi, então, calculado utilizando-se a seguinte fórmula:

Peso da porção média consumida $=\quad \frac{\text { Peso total da preparação consumida }}{\text { Número de clientes }}$
Onde:

Peso total de preparação consumida $=$ preparação produzida - (sobras + restos).

Sobras: alimentos produzidos e não distribuídos

Restos: alimentos distribuídos e não consumidos

Quadro 1. Cardápio oferecido nos 3 dias de avaliação na UAN.

\begin{tabular}{|c|c|c|c|}
\hline \multirow{2}{*}{ Refeição } & $\mathbf{0 1}$ & $\mathbf{0 2}$ & $\mathbf{0 3}$ \\
\cline { 2 - 4 } & Alface & Couve & Almeirão \\
\hline Entrada 1 & Beterraba Cozida & Tomate & Tomate \\
\hline Entrada 2 & Pepino & Feijão Branco & Repolho \\
\hline Entrada 3 & Bife de Pernil & Bife de Boi & Ovç de Frango \\
\hline Prato principal & Ovo Cozido & Ovo Cozido & Guisado de Quiabo, Chuchu e Frango \\
\hline Opção & Canjiquinha & Angu & Arroz Branco \\
\hline Guarnição & Arroz Branco & Arroz Branco & Feijão Inteiro \\
\hline Acompanhamento & Feijão Inteiro & Feijão Inteiro & Dóná \\
\hline Acompanhamento & Limão & Laranja & Paçoca \\
\hline Suco (refresco) & Doce de banana & & \\
\hline Sobremesa & &
\end{tabular}

Para avaliação nutricional do almoço de acordo com as recomendações do PAT, utilizou-se a Portaria no 193, de 5 de dezembro de 2006 [13] que estabelece que as refeições principais (almoço, jantar e ceia) deverão conter de 600 a $800 \mathrm{kcal}$, admitindo-se um acréscimo de 20\% (400 kcal) em relação ao Valor Energético Total (VET) de $2.000 \mathrm{kcal}$ por dia e deverão corresponder à faixa de 30 a 40\% do VET diário. O percentual proteico-calórico (NDPCal\%) das refeições deve ser de, no mínimo 6\% e no máximo $10 \%$ e a empresa deve oferecer, no mínimo, uma porção de frutas e uma porção de legumes ou verduras, nas refeições principais.

Para avaliação da composição nutricional do almoço utilizou-se o alimento na sua forma de consumo. Para couve, pepino, tomate, alface e repolho a avaliação foi realizada com o peso do alimento cru e os demais de acordo com a sua forma de preparo (cozido, refogado, frito). Preferencialmente, utilizou-se a Tabela Brasileira de Composição de Alimentos (TACO) [14], e caso o alimento relatado não fosse encontrado nesse material, utilizava-se outra tabela de composição de alimentos [15].

Após a avaliação do almoço dos três dias, foi feita uma média com os valores obtidos e os mesmos foram comparados com as recomendações do PAT.

Para calcular IQR utilizou-se os critérios (Quadro 2) propostos por Bandoni \& Jaime [7]. Cada componente foi avaliado e pontuado de zero a vinte, sendo que os valores intermediários foram calculados na proporção em que foram consumidos. 
Quadro 2. Descrição e critérios para pontuação mínima e máxima de cada componente do IQR.

\begin{tabular}{|c|c|c|}
\hline Componentes & $\begin{array}{l}\text { Critérios para pontuação mínima } \\
\text { (0 Pontos) }\end{array}$ & $\begin{array}{l}\text { Critérios para pontuação máxima } \\
\text { (20 Pontos) }\end{array}$ \\
\hline 1. Oferta de verduras, legumes e frutas & Oferta inferior ou igual a $80 \mathrm{~g}$ & Oferta maior ou igual a $160 \mathrm{~g}$ \\
\hline 2. Oferta de carboidratos & Oferta inferior a $40 \%$ do total de calorias & Oferta entre 55 e $75 \%$ do total de calorias \\
\hline 3. Oferta de gordura total & Oferta superior a $40 \%$ do total de calorias & Oferta entre 15 e $30 \%$ do total de calorias \\
\hline 4. Oferta de gordura saturada & $\begin{array}{l}13 \% \text { ou mais do total energia proveniente de } \\
\text { gordura saturada }\end{array}$ & $\begin{array}{l}10 \% \text { ou menos do total energia proveniente } \\
\text { de gordura saturada }\end{array}$ \\
\hline 5. Variedade do cardápio & $\begin{array}{l}4 \text { ou menos diferentes alimentos e } 2 \text { ou } \\
\text { menos grupos de alimentos que contribuíram } \\
\text { com pelo menos metade da porção do grupo } \\
\text { alimentar }\end{array}$ & $\begin{array}{l}11 \text { ou menos diferentes alimentos e } 5 \text { ou } \\
\text { menos grupos de alimentos que contribuíram } \\
\text { com pelo menos metade da porção do grupo } \\
\text { alimentar }\end{array}$ \\
\hline
\end{tabular}

O IQR foi classificado segundo Bowman et al. [16], que consideram como adequada a refeição que obtiver pontuação maior que 80 ; como refeição que precisa de melhoria aquela que obtiver pontuação entre 51 e 80 e como refeição inadequada aquela que obtiver pontuação menor ou igual a 50 .

Foi realizada uma análise descritiva dos dados por meio de frequências e medidas de tendência central.

Este estudo foi realizado após consentimento da unidade de alimentação e nutrição para o registro das quantidades de ingredientes utilizados na preparação, na pesagem dos alimentos preparados. $\mathrm{O}$ sigilo em relação ao nome da empresa foi mantido durante todas as etapas da pesquisa. E após o término do estudo, a empresa recebeu um relatório com os resultados obtidos bem como sugestões de melhoria na UAN. Foi garantido o sigilo em relação ao nome da empresa e também a devolução dos resultados encontrados.

\section{RESULTADOS}

A empresa possui um cardápio variado que atende aos funcionários administrativos e operacionais, oferecendo uma média de 380 refeições diárias. A UAN utiliza a modalidade de autoatendimento, com cardápio composto por três tipos de entradas, um prato proteico e opção de ovos, uma guarnição, arroz, feijão, doce e suco. O cliente pode se servir à vontade, havendo restrições para o consumo individual apenas para o prato proteico e doce.

Os resultados da avaliação dietética do almoço e o percentual de adequação para os nutrientes em comparação com os valores estabelecidos pelo PAT encontram-se na Tabela 1. O único nutriente que ficou abaixo da recomendação do programa foi o carboidrato. Para os demais nutrientes o almoço forneceu quantidades acima da recomendação e, convém ressaltar, que o sódio foi o nutriente que obteve maior percentual de inadequação. 
Tabela 1. Média, desvio padrão, contribuição percentual em relação ao VET, recomendação do PAT e \% de adequação dos nutrientes e do NDPCal\% do almoço de trabalhadores.

\begin{tabular}{ccccc}
\hline Nutrientes e NDPcal\% & Média \pm DP & $\begin{array}{c}\text { Contribuição (\%) } \\
\text { para o VET }\end{array}$ & $\begin{array}{c}\text { Recomendação } \\
\text { PAT }\end{array}$ & $\begin{array}{c}\text { Adequação } \\
\text { (\%) }\end{array}$ \\
\hline Energia (kcal) & $1.164,61 \pm 166,15$ & - & 600 a 800 & $145,58-194,10$ \\
Carboidrato (kcal) & $503,4 \pm 68,19$ & 43,24 & 60 & 72,07 \\
Proteína (kcal) & $264,37 \pm 73,23$ & 22,70 & 15 & 151,33 \\
Lipídio (kcal) & $387,93 \pm 119,32$ & 33,31 & 25 & 133,24 \\
Gordura saturada (kcal) & $119,67 \pm 31,21$ & 10,27 & $<10$ & 102,7 \\
Fibra (g) & $19,42 \pm 7,95$ & - & 7 a 10 & $194,3-277,43$ \\
Sódio (mg) & $1.823,57 \pm 896,96$ & - & 729 a 960 & $189,95-250,15$ \\
NDPcal\% & $13,81 \pm 3,58$ & - & 6 a 10 & $138,10-230,16$ \\
\hline
\end{tabular}

Para o IQR, a média encontrada foi de $56,53 \pm 22,92$ pontos (Tabela 2). $\mathrm{Na}$ análise dos componentes isolados, menores médias foram observadas para os carboidratos, seguidos das frutas e hortaliças.

Tabela 2. Componentes do IQR, média, desvio padrão e mediana nos três dias avaliados do almoço oferecido aos trabalhadores.

\begin{tabular}{lrrrcc}
\hline \multicolumn{1}{c}{ Classificação do IQR } & 1o dia & 2o dia & 3o dia & Média \pm DP & Mediana \\
\hline Indicador de frutas e hortaliças & 8,00 & 0,00 & 12,50 & $6,83 \pm 6,33$ & 8,00 \\
Indicador de carboidratos & 10,39 & 4,91 & 0,00 & $5,1 \pm 5,20$ & 4,61 \\
Indicador de gorduras & 20,00 & 20,00 & 0,00 & $13,33 \pm 11,55$ & 20,00 \\
Indicador de gorduras saturadas & 20,00 & 20,00 & 0,00 & $13,33 \pm 11,55$ & 20,00 \\
Indicador de variedade & 17,53 & 17,53 & 18,73 & $17,93 \pm 0,69$ & 17,53 \\
\hline Índice de Qualidade da Refeição & 75,92 & 62,44 & 31,23 & $56,53 \pm 22,92$ & 62,44 \\
\hline
\end{tabular}

\section{DISCUSSÃO}

Este estudo possibilitou a avaliação do almoço oferecido por uma empresa cadastrada no PAT, segundo as recomendações estabelecidas pelo programa e ainda pelo IQR.

O valor energético total do almoço encontrado foi de 1.164,61£166,15 Kcal, o qual está acima da recomendação (600 a $800 \mathrm{Kcal}$ ), revelando assim, ingestão excessiva de energia nos almoços oferecidos aos trabalhadores. Sendo assim, com relação ao valor energético, este se encontra superior ao valor estabelecido pelo programa, o que poderia contribuir para desenvolvimento de sobrepeso e obesidade, o que já foi descrito em outros estudos [15, 17]. Ressalta-se, entretanto, que o gasto energético dispendido nas atividades desempenhadas pelos trabalhadores não justifica esse alto consumo, pois, os mesmos desempenham atividade física moderada [?].

O teor de sódio do almoço superou a recomendação preconizada em $89,95-150,15 \%$ a mais. Cabe ressaltar que essa quantidade de sódio é atribuída somente ao sal utilizado para o preparo da refeição e ao sódio dos alimentos. Não foi computado o sódio referente ao sal de adição, ou seja, o distribuído em 
saleiros sobre as mesas, que cada usuário poderia utilizar no momento da refeição e, portanto, a ingestão do nutriente pode ter sido subestimada, o que é preocupante à saúde dos usuários da UAN estudada. A ingestão elevada de sódio é observada em outros estudos envolvendo empresas cadastradas pelo PAT. Em Suzano-SP, Salas et al. [18] encontraram um teor médio de sódio no almoço de $2.435 \mathrm{mg}$. De acordo com Spinelli \& Koga [19], a hipertensão arterial é observada principalmente em comunidades com alta ingestão de sal. Essa constatação parece ser independente de outros fatores de risco, como obesidade e alcoolismo.

Dados do Instituto Brasileiro de Geografia e Estatística ${ }^{[6]}$ revela que o consumo de sal do brasileiro está em 12 gramas diários. A Organização Mundial de Saúde ${ }^{[20]}$ definiu a recomendação em menos de $5 \mathrm{~g}$ de cloreto de sódio ou sal de cozinha (que corresponde a menos que $2 \mathrm{~g}$ de sódio) como a quantidade máxima saudável para ingestão alimentar diária. Entretanto, o consumo médio do brasileiro é superior ao recomendado, o que corrobora com os dados encontrados no presente estudo. Vale ressaltar que o consumo elevado de sal é um fator de risco para desenvolvimento de hipertensão e este constitui como uma importante causa de incapacidade temporária dos trabalhadores ${ }^{[21]}$.

Considerando que a prevalência estimada de hipertensão no Brasil atualmente é de $35 \%$ para a população acima de 40 anos [22], são necessárias implementações de ações que conduzam a uma diminuição da ingestão de sódio pela população estudada. De acordo com a "Estratégia Global sobre Alimentação, Atividade Física e Saúde”, da WHO [11], as empresas que fornecem alimentação coletiva devem adotar iniciativas para redução da quantidade de gorduras, açúcar e sal dos alimentos, elaborando opções inovadoras e saudáveis nos cardápios. Dessa forma, a restrição de sal na dieta é uma medida recomendada para a população de um modo geral e pode também reduzir em longo prazo o risco de problemas cardiovasculares.

Para carboidratos, a quantidade oferecida estava aquém da recomendação e, em contrapartida, a oferta de proteínas e lipídeos estavam acima da recomendação. Assim, o almoço foi caracterizado como hipoglicídico, hiperprotéico e hiperlipídico. Os resultados encontrados no presente estudo se assemelham aos encontrados por Geraldo et al. ${ }^{[17]}$ no qual foram encontrados os seguintes valores: $50,95 \%$ de carboidratos, $18,85 \%$ de proteínas e 30,18\% de lipídeos. Jaime et al. [0] também avaliaram 72 empresas cadastradas no PAT em São Paulo e verificaram que a maioria dos cardápios oferecia gordura e proteína em excesso e carboidrato em deficiência. Cabe ressaltar que o carboidrato é o nutriente que deve contribuir com $60 \%$ do total de energia ingerida ${ }^{[1]}$ e baixa ingestão de alimentos fontes deste macronutriente promove um desequilíbrio na proporção dos outros macronutrientes ingeridos, além de onerar o custo da refeição. Além disso, a alta ingestão de lipídios pode conduzir a distúrbios no metabolismo lipídico e predispor ao desenvolvimento de doenças cardiovasculares [11, 23].

A média para NDPcal\% encontrada no presente estudo está acima da recomendação do PAT, o que foi observado também por Savio et al. ${ }^{[24]} \mathrm{em}$ estudo realizado com 1.044 clientes de 52 UANs, em que o NDPcal $\%$ médio foi de $16 \%$, superior à recomendação. Vale ressaltar, que a metodologia para cálculo do NDPCal\% em ambos estudos foi por meio do cálculo a partir do consumo real e não pelo cardápio planejado. Um valor para NDPCal\% acima do limite superior estabelecido caracteriza uma refeição hiperproteica e com custo elevado. Do ponto de vista nutricional uma ingestão excessiva de proteína é desnecessária, já que o organismo utilizará a proteína como fonte calórica e não para síntese proteica ${ }^{[25]}$.

O resultado encontrado para consumo de gordura saturada também foi além da recomendação proposta pelo PAT e as preparações que contribuíram para este resultado foram: bife de pernil, bife bovino e linguiça de frango. Outros estudos que avaliaram as refeições de empresas cadastradas no PAT também encontraram cardápios que ofereciam excesso de gordura saturada [5, 24], corroborando com os achados do presente estudo. Esses achados são preocupantes, pois o consumo excessivo de gorduras saturadas está relacionado a várias doenças crônicas não transmissíveis, tais como doenças cardiovasculares, diabetes, obesidade, acidentes cerebrovasculares e câncer [23].

Outros trabalhos que avaliaram o consumo alimentar fora de casa, também indicam que a inadequação na ingestão de alimentos não é apenas uma realidade nas empresas cadastradas no PAT, mas um padrão de comportamento alimentar. Há evidências que a alimentação fora de casa possui maior densidade energética, com maiores quantidades de gordura, gorduras saturadas e menor quantidade de micronutrientes $[26,27]$. 
Para fibras, o valor médio encontrado $(19,42 \mathrm{~g}$ $\pm 7,95 \mathrm{~g})$ superou a recomendação estabelecida pelo programa para as refeições principais. O consumo de feijão foi relevante para a oferta de fibras, contribuindo com uma média de $12,49 \mathrm{~g}(64,31 \%$ do total $)$ e, além disso, houve oferta de hortaliças nas entradas e nas guarnições. Savio et al. [24] encontraram consumo mediano de fibras inferior ao presente estudo devido ao baixo consumo de hortaliças cruas e da presença de guarnições sem utilização de hortaliças. Sabe-se que o consumo de fibras é importante para a saúde, pois entre outros benefícios, contribuem para o bom funcionamento do trato gastrintestinal, além de auxiliar na redução da absorção do colesterol pelo organismo e manutenção dos níveis glicêmicos [25]. Entretanto, a UAN estudada não oferece nenhuma porção de frutas no almoço, sendo que de acordo com a Portaria no 193, de 5 de dezembro de 2006 [13], os cardápios deverão oferecer, pelo menos, uma porção de frutas nas refeições principais, pois, além de serem fontes de fibras, contribuem com a ingestão de vitaminas e minerais.

A média do IQR encontrado para o almoço $(56,53)$ se assemelha ao índice de 56,23 encontrado por Bandoni \& Jaime [3] para empresas de pequeno porte em São Paulo, demonstrando que a qualidade da alimentação não difere muito de outras regiões.

A pontuação encontrada classifica o almoço servido aos trabalhadores como "refeição que precisa de melhoria", o que demonstra a necessidade de intervenção para a adequação da alimentação com vistas ao atendimento do que é estabelecido pelo Guia Alimentar para a População Brasileira [23], pela Diretriz Brasileira de Hipertensão ${ }^{[2]}$ e WHO ${ }^{[1]}$ para prevenção de doenças crônicas não degenerativas e promoção da saúde.

Ressalta-se que diante das constantes modificações no quadro de morbimortalidade no país e atualizações das recomendações nutricionais torna-se necessária uma revisão do PAT.

\section{CONCLUSÕES}

Os resultados obtidos estão em concordância com outros estudos [3, 18, 26] e mostra que o almoço oferecido pela UAN não estava adequado de acordo com os parâmetros do Programa de Alimentação do Trabalhador e o IQR. Dessa forma, pode propiciar inadequação nutricional para os trabalhadores, contrariando os objetivos primordiais do PAT que é a promoção da saúde. Torna-se necessário a inclusão de ações como medidas educativas de intervenção alimentar com envolvimento dos gestores e nutricionistas para adequação nutricional das refeições. Faz-se necessária também a implantação de programas de educação nutricional para os usuários, principalmente, quando se utiliza o sistema de distribuição self service, objetivando o empoderamento dos mesmos acerca de promoção de saúde. Essas ações poderão contribuir para uma alimentação adequada, a qual refletirá na redução do quadro que o país hoje vivencia de aumento das doenças que tem como fator desencadeante importante hábitos alimentares inadequados.

\section{REFERÊNCIAS}

[1] Brasil, Ministério do Trabalho e Emprego. Programa de alimentação do trabalhador: legislação. 4a ed. Brasília (DF); 2001.

[2] Brasil. Ministério do Trabalho e Emprego. Programa de alimentação do trabalhador [internet]. 2008 [citado em 09 jun 2015]. Disponível em: http:// pat.mte.gov.br/relatorios2008

[3] Freire RB, Salgado RS. Avaliação de cardápios oferecidos a trabalhadores horistas. Mundo Saúde. 1998;22(5):198-301.

[4] Garcia RWD. Reflexos da globalização na cultura alimentar: considerações sobre as mudanças na alimentação urbana. Rev Nutr. 2003;16(4):483-92.

[5] Gorgulho BM, Lipi M, Marchioni DML. Qualidade nutricional das refeições servidas em uma unidade de alimentação e nutrição de uma indústria da região metropolitana de São Paulo. Rev Nutr. 2011;24(3):463-472.

[6] Instituto Brasileiro de Geografia e Estatística - IBGE. Pesquisa de Orçamentos Familiares (POF) 2008-2009: análise da disponibilidade domiciliar de alimentos e do estado nutricional no Brasil. Rio de Janeiro; 2010.

[7] Bandoni DH, Jaime PC. A qualidade das refeições de empresas cadastradas no Programa de Alimentação do Trabalhador na cidade de São Paulo. Rev Nutr. 2008;21(2):177-184.

[8] Bandoni DH, Brasil BG, Jaime PC. Programa de Alimentação do Trabalhador: representações sociais de gestores locais. Rev Saúde Pública. 2006;40(5):837-842.

[9] Jaime PC, Bandoni DH, Geraldo APG, Rocha RV. Adequação das refeições oferecidas por empresas cadastradas 
no programa de alimentação do trabalhador na cidade de São Paulo. Mundo Saúde. 2005;29(2):186-191.

[10] Chu C, Breucker G, Harris N, Stitzel A, Gan X, Gu X, Dwyer S. Health promoting workplaces - interational settings development. Health Promot Int. 2000;15(2):155-167.

[11] World Health Organization. Global Strategy on diet, physical activity and health. Food Nutr Bull. 2004;25(3):292302.

[12] Abreu ES, Spinelli MGN, Pinto AMS. Gestão de Unidades de Alimentação e Nutrição: um modo de fazer. 2a ed. São Paulo: Metha; 2007.

[13] Brasil. Ministério do Trabalho e Emprego. Portaria Interministerial no 66, de 25 de Agosto de 2006. Altera os parâmetros nutricionais do Programa de Alimentação do Trabalhador - PAT. Diário Oficial da União. 18 ago 2006.

[14] TACO - Tabela Brasileira de Composição dos Alimentos. Campinas: Ed. Nepa-Unicamp; 2006 [acesso em 22 set 2015]. Disponível em: http://www.unicamp.br/nepa/taco/contar/taco_4_edicao _ampliada_e_revisada

[15] Philippi ST. Tabela de composição de alimentos: suporte para decisão nutricional. 2a ed. Brasília (DF); 2002.

[16] Bowman SA, Lino M, Gerrior SA, Basiotis PP. The healthy eating index: 1994-1996. Washington (DC): Departament of Agriculture, Center for Nutrition Policy and Promotion; 1998.

[17] Geraldo APG, Bandoni DH, Jaime PC. Aspectos dietéticos das refeições oferecidas por empresas participantes do Programa de Alimentação do Trabalhador na Cidade de São Paulo, Brasil. Rev Panamer Salud Publica. 2008;23(1):1925.

[18] Salas CKTS, Spinelli MGN, Kawashima LM, Ueda AM. Teores de sódio e lipídios em refeições almoço consumidas por trabalhadores de uma empresa do município de Suzano, SP. Rev Nutr. 2009;22(3):331-339.
[19] Spinelli MGN, Koga T. Avaliação do consumo de sal em uma unidade de alimentação e nutrição. Nutrire 2007;32(2):15-27.

[20] World Health Organization. Guideline: Sodium intake for adults and children [internet]. Geneve, 2012. [acesso em 22 set 2015]. Disponível em: http://www.who.int/nutrition/publications/guidelines

[21] IV Diretrizes Brasileiras de Hipertensão. Arq Bras Cardiol. 2010;95(Supl 1):1-51.

[22] Brasil. Ministério da Saúde. Sal aumenta a pressão. Ninguém merece trabalhar sob pressão [internet]. 2015 [citado em 01 ago 2015]. Disponível em: http://portal.saude.gov.br/portal/saude/visualizar_texto.cf m?idtxt=23616

[23] Brasil. Ministério da Saúde. Secretaria de Atenção à Saúde. Guia alimentar para a população brasileira: promovendo a alimentação saudável [internet]. Brasilia; 2005. Série A: normas e manuais técnicos [citado em 28 jan 2015]. Disponível em: http://bvsms.saude.gov.br/bvs/publicacoes/guia_alimentar _populacao_brasileira.pdf

[24] Savio KEO, Costa THM, Miazakib E, Schmitz BAS. Avaliação do almoço servido a participantes do programa de alimentação do trabalhador. Rev Saúde Pública. 2005;39(2):148-155.

[25] Costa NMB, Peluzio MCG. Nutrição Básica e Metabolismo. Viçosa: Editora UFV; 2008.

[26] Kearney JM, Hulshof KF, Gibney MJ. Eating patterns: temporal distribution, converging and diverging foods, meals eaten inside and outside of the home: implications for developing FBDG. Public Health Nutr. 2001;4(2B):693-698.

[27] Orfanos P, Naska A, Trichopoulos D, Slimani N, Ferrari P, Van Bakel M, et al. Eating out of home and its correlates in 10 European countries. The European Prospective Investigation into Cancer and Nutrition (EPIC) study. Public Health Nutr. 2007;10(12):1515-1525. 